\title{
THE PULLED ARRAY TRANSIENT ELECTROMAGNETIC METHOD
}

\author{
Sørensen, K.I., Department of Earth Sciences, \\ Aarhus University, Finlandsgade 8, DK-8200 Aarhus N, Denmark \\ Tlf $+4586161666-$ Fax +4586101003
}

\section{INTRODUCTION}

The mapping of subsurface resistivity structures using transient electromagnetic soundings, TEM, has gained an important role in hydrogeological investigations in Denmark. Regional mapping with TEM have produced results, which leaves no doubt as to the ability of the method to delineate freshwater sandy aquifers in well conducting hosts of Tertiary clays. These results have been achieved measuring in a dense grid and do demonstrated the necessity of estimating the data quality and variation by correlating adjecent data sets to reveal distortion and multidimensionality of the measurements. The fact that these investigations are often carried out in densely populated areas, where the cultural noise level is high, accentuates this necessity even more (Christensen and Sorensen, 1994).

In this context a new method, the Pulled Array Transient Electromagnetic Method, PATEM, is developed. The method applies a transmitter and a receiver coil system which are towed along profile lines while measuring. With this method the cost of the field work is drastically reduced. Furthermore, as the measurements are recorded very densely, adjacent data sets can be correlated to ensure good data quality, and distortion from cultural effect from powerlines, fences, etc. of the data sets is more easily revealed. Finally the necessary density of the data sets for multidimensional interpretation can be provided (Auken, 1995).

\section{TRANSIENT ELECTROMAGNETIC SOUNDINGS IN DENMARK}

In the initial phase of the application of the transient method in Denmark it became clear that the distortion from "cultural conductors", such as powerlines, cables, steel pipes, metal fences and electromagnetic noise, affects the measurements seriously. Most of these conductors in Denmark are mapped by the authorities, but it is a comprehensive task to form an overview from the overwhelming number of files. Furthermore, not all of the conductors are mapped (i.e. metal fences), especially in the farmland. Another problem is the lack of a general rule of thumb for a minimum distance to disturbing elements which will ensure the distortion of the measurement to be negligible. Finally, when multidimensional interpretations are demanded dense data collection are required (Christensen, 1995). These circumstancies all demand spatial dense measurements.

Therefore, since a trade-off exists between cost of work and reliability of measurements, it has become a general practice to perform $15-30$ soundings pr. $\mathrm{km} 2$. This number of soundings may provide a reasonable background for estimating whether data are reliable by correlating interpretations of nearby data sets and compare the variations with the knowledge of the geology, but there is no doubt that a denser grid will indeed increase the quality of the investigation. 
Large areas have been investigated with TEM in order to get detailed information on the regional aquifers. The field work involved has indeed been comprehensive and cumbersome. There is no doubt that if the transient method shall have a future as a mapping tool of aquifers in cultivated areas it is necessary to develop a faster field technique in order to meet the requirements of measuring in dense grids (Sorensen et al., 1995).

In this context the development of the PATEM method was initiated.

\section{THE PATEM METHOD}

A transmitter and receiver system has been developed to investigate the experimental design of the PATEM method (Sorensen et al., 1995).

The transmitter system is optimised to transmit high currents with short turnoffs in small coils of the order of 2.5 times 2.5 meters. Furthermore each coil is constructed in coil segments each with its own transmitter in order to minimise turnoff time and still maintain an large moment.

The receiver coil system consists of a suspended horizontal coil with parallel amplifier channels with progressive gain Furthermore, the channels have a adjustible analog time gate pattern in order to diminish quantification noise. This parallel segmented recording is necessary in order to cover the dynamic range of the secondary field. The analog receiver circuits transmit their digitised signals by optics to a processing unit (a powerful PC). This setup ensures an optimal signal to noise ratio in the demanded dynamic range. The data series are recorded and stored separately for each transmitter cycle. The data processing is performed on a chosen number of measured series. In order to obtain a sufficient signal to noise ratio, especially at late delay times, a digital synchronous and predictive filtering technique is introduced. Beside the data processing and the electronic hardware initiatives much effort has been put into practical implementations and field logistics.

It is not possible, in a single transmitter coil system, to obtain sufficient magnetic moment to be able to measure the late time secondary field and to ensure, that the influence from the current turnoff in the transmitter coils does not affect the recording of the early time secondary field. Hence a segmented transmitter system is applied. Transmitter coils with fast turnoff and small moment are used for the recording of early time decays and coils with large moment and slow turnoff for recording late decays. These series are combined to form a complete dataset for the entire time range (10 microseconds to 10 milliseconds).

The comparison with traditional instruments (PROTEM 47) show excellent agreement, and repetitive measurements with the equipment demonstrate the repeatability of the method.

\section{RESULTS}

The instrumentation has been preliminary tested at the Vestereng testsite north of Aarhus. The design of the moveable receiver coil system is still under development in order to gain further supression of the noise related to the coil movement. The obtained results so far are very promising. Figure 1 shows the noise pick-up from the reciever coil system in movement and in rest. It can be observed that the noise level with the coil in movement is approximately twice that of the coil in rest.

The design of the transmitter coil is still in development in order to achieve a design that is optimal for field operation. The present design applying two different transmitter moments 


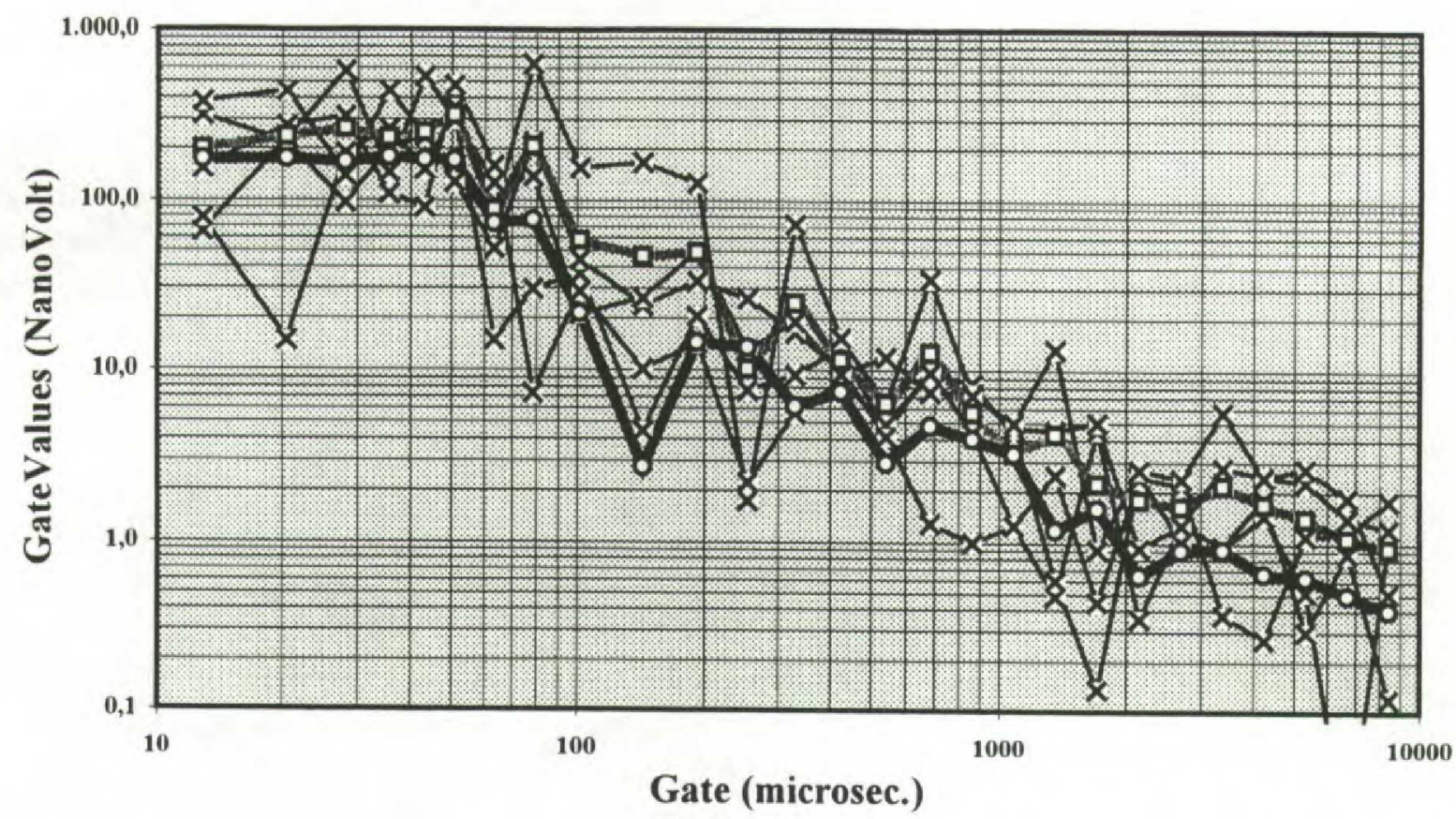

Figure 1: Noise $\mathrm{dB} / \mathrm{dt}$ signals from reciever coil. The shown data are the average of 500 transmitter cycles $(50 \mathrm{~Hz}$ rep.). The lower thick solid curve are the noise data when the coil is in rest and the thin curves with crosses are the different noise data from the coil in movement ( $2 \mathrm{~km}$ per hour) while measuring. The upper gray thick curve are the average of the noise data from the coil in movement.

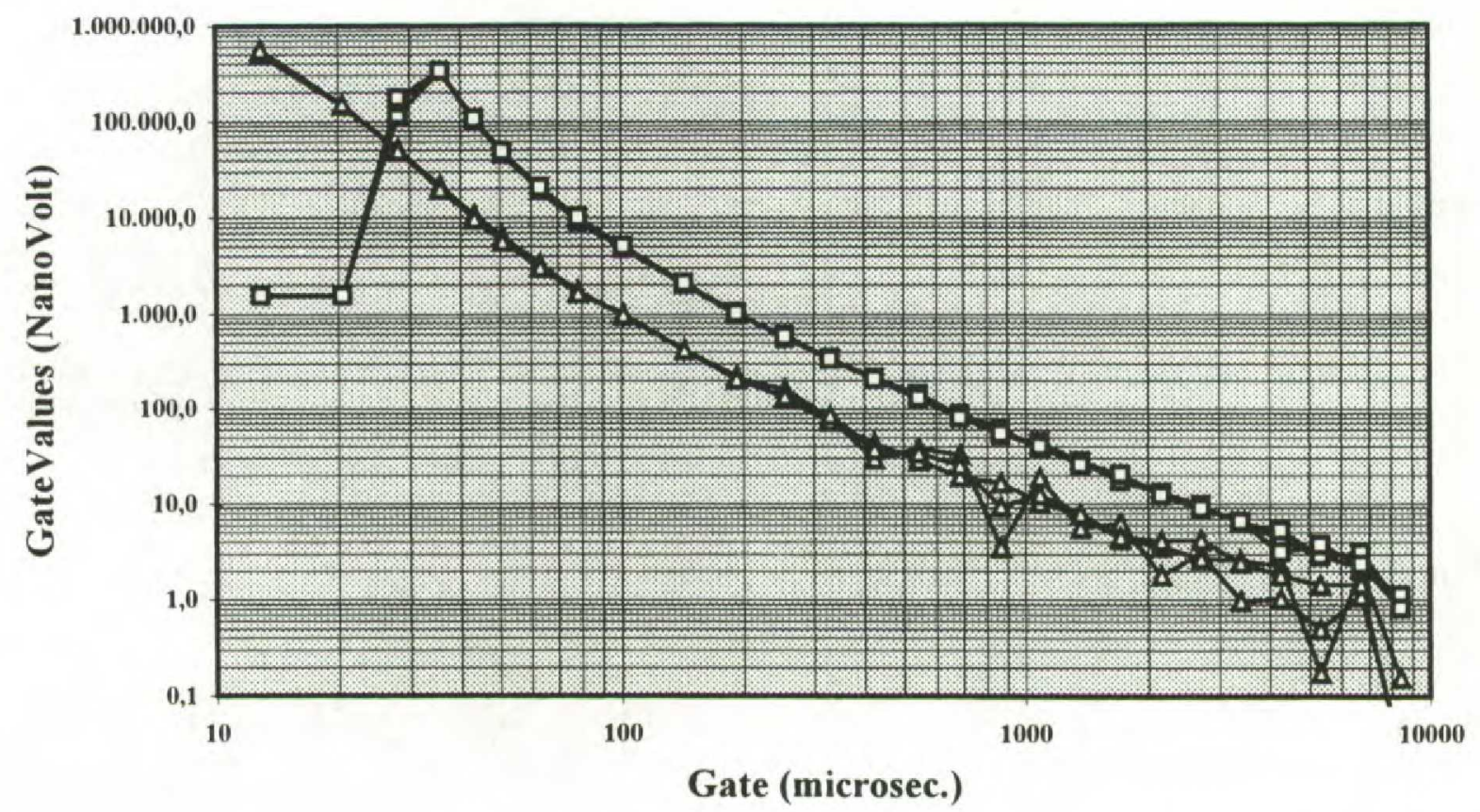

Figure 2: Measured $\mathrm{dB} / \mathrm{dt}$ decay signals from the coil systems in movement $(2 \mathrm{~km}$ per hour) while measuring. The upper series uses a transmitter moment of $3150 \mathrm{~A} \mathrm{x} \mathrm{m} 2$ and the lower more noise series uses a transmitter moment of $700 \mathrm{~A} \mathrm{x} \mathrm{m2}$. The early time gates (up to 40 microseconds) of the upper series are controlled by the primary field from the current turnoff in the transmitter coils. 
of 700 Amp x m2 and 3150 Amp x m2 show remarkable results obtained in a typical danish geological setting as the Vestereng testsite. Measurements not influented by noise are obtained to gates with center times from 10 microseconds up to 8 to 10 milliseconds applying the combined transmitter system and the receiver coil system in movement while measuring. Figure 2 demonstrates the results obtained along the same profile as the noise measurements shown in figure 1. It is observed, that a combination of the decays obtained from small transmitter moment and from large moments (interchange at approximately 200 microseconds) will provide the entire decay of the magnetic field in the range from 10 microseconds to 8 milliseconds.

\section{CONCLUSION}

An instrumentation has been developed for performing Pulled Array Continuous Electromagnetic soundings, PATEM. Investigations have been carried out to show that the proposed design is realisable. Small movable transmitter coils with segmented transmitter technique are applied to cover the full delay time range. The receiver system utilises parallel segmented recording with analog adjustable time gates of the signals in order to cover the dynamic range of the secondary fields, and a digital postprocessing of the stored data from each transmitter cycle is applied to enhance the signal to noise ratio. The obtained results are very promising.

PA-TEM enables large scale TEM surveys with closely spaced profile lines to be carried out with a field efficiency which cannot be provided by traditional instrumentation, and in areas with high cultural activities the method can provide the necessary information about cultural distortion and multidimensionality of the measurement by correlation of nearby data sets.

\section{REFERENCES}

Auken, E., 1995: 1D Time Domain Electromagnetic Interpretations over 2D/3D Structures. Proceedings of the Symposium on the Application of Geophysics to Engineering and Environmental Problems, Orlando, April 1995, p 329-338.

Christensen, N.B \& Sørensen, K.I.,1994: Integrated use of electromagnetic methods for hydrogeological investigations. Proceedings of the Symposium on the Application of Geophysics to Engineering and Environmental Problems, Boston, March 1994, p 163-176.

Christensen, N.B., 1995: Imaging and inversion of transient electromagnetic soundings.Proceedings of the Symposium on the Application of Geophysics to Engineering and Environmental Problems, Orlando, April 1995, p 511-517

Sørensen, K.I., Effersø F. and Christensen, A. J., 1995: Pulled Array Transient Electromagnetic Method, PA - TEM. Proceedings of the Symposium on the Application of Geophysics to Engineering and Environmental Problems, Orlando, April 1995, p 899-903

Sørensen, K.I. et. al, 1995: Water Supply, Department of Earth Sciences, Aarhus University, 1995, pp 24. 